CASE REPORT

\title{
F-18 Fluorodeoxyglucose (FDG) Negative Hepatoblastoma with High Alp- haphetoprotein Levels; Better Prognosis
}

\author{
Koç $\mathrm{ZP}^{1}$, Kara $\mathrm{PO}^{1}$, Çıtak $\mathrm{EC}^{2}$ and Esen $\mathrm{K}^{3}$ \\ ${ }^{1}$ Nuclear Medicine Department, Mersin University, Mersin, Turkey \\ ${ }^{2}$ Oncology Department, Mersin University, Mersin, Turkey \\ ${ }^{3}$ Department of Radiology, School of Medicine, Mersin University, Mersin, Turkey
}

*Corresponding author: Koç ZP, MD, Associate Professor, Nuclear Medicine Department, Mersin University, 33343 Mersin, Tel: 903242410000 , Fax: 903242410098, Email: zehrapinarkoc@gmail.com

Citation: Koç ZP, Kara PO, Çitak EC, Esen K (2018) F-18 Fluorodeoxyglucose (FDG) Negative Hepatoblastoma with High Alphaphetoprotein Levels; Better Prognosis. J Pediatr Dis Neonatal Care 1: 105

Article history: Received: 19 March 2018, Accepted: 22 May 2018, Published: 24 May 2018

\begin{abstract}
Although hepatoblastoma is the most common malign tumor in childhood in the liver there are few studies including fluorodeoxyglucose positron emission tomography/computed tomography imaging and hepatoblastoma. The patient included in this report has multiple hepatic lesions with high alphaphetoprotein (AFP) levels suggestive of hepatoblastoma but no increased FDG uptake in the liver lesions who responded the chemotherapy very well.
\end{abstract}

Keywords: Hepatoblastoma; Fluorodeoxyglucose; Positron Emission Tomography; Alphaphetoprotein

\section{Introduction}

The hepatoblastoma is the primary malignant tumor of liver of childhood, most frequently in children at age of $<5$ years old [1]. The tumors arises from the embryonic origin thus is characterized with high alphaphetoprotein levels and might show different characteristics in FDG positron emission tomography/computed tomography (PET/CT) imaging [2,3]. The first studies of FDG PET/CT and hepatoblastoma showed that although PET might be helpful in the determination of recurrences of the hepatoblastoma; careful interpretation is necessary due to the false positive results [3]. The histopathology of the liver tumor of the case in this study confirmed the diagnosis of the hepatoblastoma. The patient in this case report is a good example of a good responder hepatoblastoma who has negative metabolic imaging and elevated alphaphetoprotein level which might point out a good prognosis however this issue needs to be evaluated by future series.

\section{Case report}

Eighteen months old male patient presented with abdominal distension for two-three months attended to the hospital. The informed consent of the patients' family was obtained. Ultrasonography revealed gross liver tumor involving right liver lobe and elevated

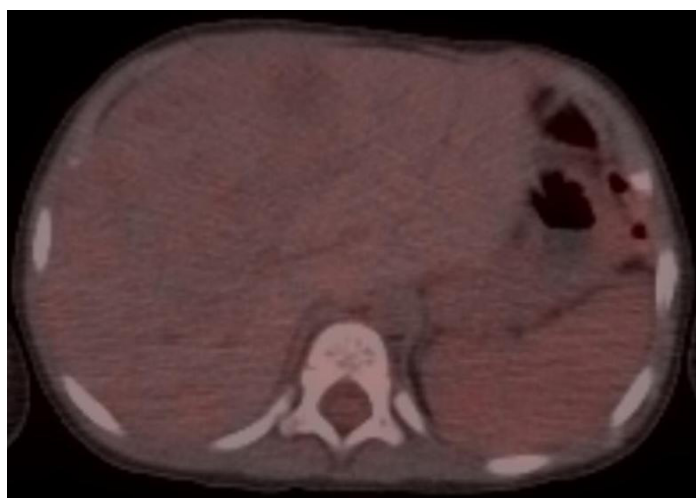

Figure 1a: Transaxial projection image of the F-18 FDG PET/CT corresponding

to the gross liver lesion without pathological FDG accumulation 


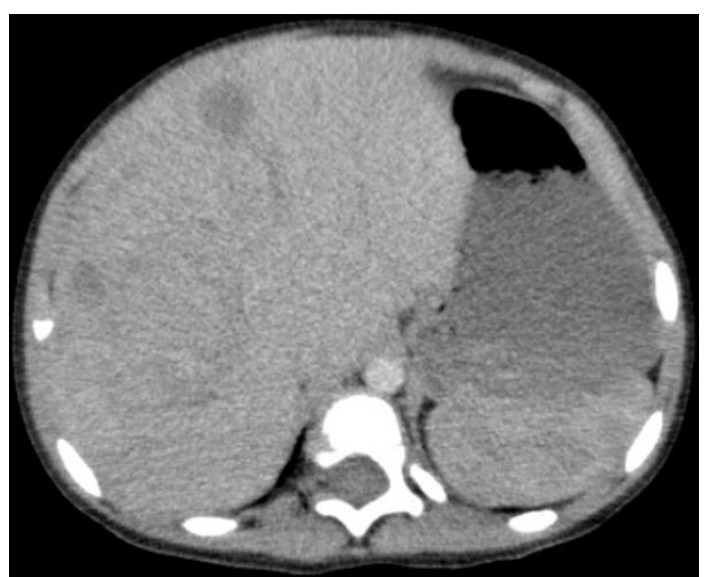

Figure 1b: The transaxial section of the contrast enhanced CT images showing the same liver lesion

alphaphetoprotein levels (1745 IU/mL). The patient was referred to F-18 FDG PET/CT study for metabolic characterization. PET/ $\mathrm{CT}$ imaging was performed after approximately $2 \mathrm{mCi}(74 \mathrm{MBq})$ intravenous injection of F-18 FDG and 4 hour fasting period. The imaging was performed approximately 1 hour after administration of the radiopharmaceutical in craniocaudal direction as a whole body acquisition. PET/CT imaging revealed liver mass without significant FDG acculumation (Figure 1). The patient received chemotherapy and responded very well with significant decrease in AFP levels in two month follow up $(91.93 \mathrm{IU} / \mathrm{mL})$.

\section{Discussion}

Hepatoblastoma is one of the malign tumors of childhood age which might metastasize to abdomen, lung or brain [4]. Usually the hepatoblastoma accumulate FDG. Mody et al. showed FDG accumulation in the all 5 of their patients with hepatoblastoma [4]. Previous studies also showed that FDG accumulation might help to differentiate hepatoblastoma from benign lesions $[5,6]$.

FDG PET/CT also is a documented diagnostic tool in recurrence evaluation in the patients with hepatoblastoma [7]. Previously radiopharmaceutical like Ga-67, Tl-201 and Tc-99m labeled alphaphetoprotein were used in the determination of the hepatoblastoma [8-10]. Fluorocholine PET/CT is another discriminative method in the evaluation of hepatic tumors which is considered to be very useful in the differentiation of hepatoblastoma from benign lesion, staging and treatment response in previous series [11]. Previous researchers have pointed out the importance of the metabolic characterization [6]. In a previous case report the omental and liver recurrences was found by the FDG PET/CT and successfully removed surgically in a patient with elevation of AFP levels withoutsuccessful localization of the lesion by CT and MR [12]. In the restaging of the patients with hepatoblastoma; a previous study showed 100\% agreement with the histopathology results and the FDG PET/CT findings [13]. Previous researchers do not consider FDG PET/CT in the initial diagnosis but found valuable in the early recurrence evaluation [14,15]. Cistaro, et al., have showed significantly better results of the PET/CT than CT/MR (100\% versus 44\%) [13]. A certain type of hepatoblastoma; pure fatal subtype has minimal mitotic activity and has lower FDG affinity and thus limited efficiency of FDG PET/CT in the staging [16]. In other series the diagnostic specificity of AFP levels were higher than PET/CT or CT imaging however high risk patients with low AFP levels $(<100 \mathrm{ng} / \mathrm{ml})$ were considered to be the poor responders and worse outcome patients $[17,18]$. Recently F-DOPA PET/ CT has shown high diagnostic accuracy in a patient with scapular metastasis thus promising results for the hepatoblastoma [19].

Although both FDG PET/CT and AFP levels have prognostic information regarding the hepatoblastoma; combination of these parameters has not been reported before. According to this case report negative FDG PET/CT examination with high AFP levels might predict good prognosis and response to chemotherapy.

\section{Reference}

1. Herzog CE, Andrassy RJ, Eftekhari F (2000) Childhood cancers: hepatoblastoma. Oncologist 5: 445-53.

2. Clericuzio CL, Chen E, McNeil DE, O'Connor T, Zackai EH, et al. (2003) Serum alpha-fetoprotein screening for hepatoblastoma in children with BeckwithWiedemann syndrome or isolated hemihyperplasia. J Pediatr 143: 270-2.

3. Wong KK, Lan LC, Lin SC, Tam PK (2004) The use of positron emission tomography in detecting hepatoblastomarecurrence--a cautionary tale. J Pediatr Sur 39: $1779-81$

4. Mody RJ, Pohlen JA, Malde S, Strouse PJ, Shulkin BL (2006) FDG PET for the study of primary hepatic malignancies in children. Pediatr Blood Cancer 47: 51-5. 5. Kurtaran A, Becherer A, Pfeffel F, Müller C, Traub T, et al. (2000) 18F-fluorodeoxyglucose (FDG)-PET features of focal nodular hyperplasia (FNH) of the liver. Liver 20: 487-90.

6. Magini G, Farsad M, Frigerio M, Serra C, Colecchia A, et al. (2009) C-11 acetate does not enhance usefulness of F-18 FDG PET/CT in differentiating between focal nodular hyperplasia and hepatic adenoma. Clin Nucl Med 34: 659-65.

7. Philip I, Shun A, McCowage G, Howman-Giles R (2005) Positron emission tomography in recurrent hepatoblastoma. Pediatr Surg Int 21: $341-5$.

8. Howman-Giles R, Bernard EJ, Uren R (1997) Pediatric nuclear oncology. Q J Nucl Med 41: 321-35. 
9. Bernard EJ, Nicholls W, Howman-Giles R, Kan A, Stevens M (1997) Tl201 positive, Ga-67 negative hepatoblastoma: a case report of a 12 years old boy. Clin Nucl Med 22: 835-7.

10. Kairemo KJ, Lindahl H, Merenmies J, Föhr A, Nikkinen P, et al. (2002) Anti-alpha-fetoprotein imaging is useful for staging hepatoblastoma. Transplantation 73: 1151-4.

11. Bieze M, van Gulik TM, Bennink RJ (2013) Hepatoblastoma evaluated by 18F-Fluoromethyl Choline PET/CT. Clin Nucl Med 38: 80-82.

12. Figarola MS, McQuiston SA, Wilson F, Powell R (2005) Recurrent hepatoblastoma with localization by PET/CT. Pediatr Radiol 35: 1254-8.

13. Cistaro A, Treglia G, Pagano M, Fania P, Bova V, et al. (2013) A comparison between 18F-FDG PET/CT imaging and biological and radiological findings in restaging of hepatoblastoma patients. Biomed Res Int 709037.

14. Patel CD, Kumar R (2007) Positron emission tomography and positron emission tomography-computerized tomography in pediatric patients. J Indian Assoc Pediatr Surg 12: 120-4.

15. Yang WT, Johnson PJ (1999) Monitoring response to treatment in liver tumours. Baillieres Best Pract Res Clin Gastroenterol 13: 637-54.

16. McCarville MB, Roebuck DJ (2012) Diagnosis and staging of hepatoblastoma: imaging aspects. Pediatric Blood \& Cancer 9: 793-9.

17. Rojas y, Guillerman RP, Zhang W, Vasudevam SA, Nuchtern JmG, et al. (2014) Relapse surveillance in AFP-positive hepatoblastoma: re-evaluating the role of imaging. Pediatr Radiol 44: 1275-80.

18. Semraro M, Branchereau S, Maibach R, Zsiros J, Casanova M, et al. (2013) Relapses in hepatoblastoma patients: clinical characteristics and outcome - experience of the International Childhood Liver Tumour Strategy Group (SİOPEL). Eur J Cancer 49: 915-22.

19. Zhang B, He Q, Shi X, Wang X, Zhang X (2017) Recurrent scapular metastasis from Hepatoblastoma shown on FDG PET/CT and F-DOPA PET/CT. Clin Nucl Med 42: e449-51. 